\title{
Aprovechamiento de la política pública en el fomento de la empresa familiar
}

\section{Use of public policy in promoting family business}

\author{
Edwin Santamaría Freire * \\ edwinjsantamaria@uta.edu.ec \\ Klever Moreno Gavilanes \\ kleveramoreno@uta.edu.ec \\ $* *$ \\ Edwin Santamaría Díaz \\ ec.santamaria@uta.edu.ec
}

\begin{abstract}
Resumen
La política de promoción de las empresas busca la explotación de las potencialidades del país, por ello se planteó como objetivo de este estudio: Determinar el aprovechamiento de las políticas públicas en el crecimiento de la empresa familiar. La investigación de tipo descriptiva fue aplicada a doscientos doce empresas familiares y se encontró como resultados qué: Las empresas familiares están administradas por miembros de la familia, especialmente el fundador, con una capacidad de mejorar la calidad de vida de sus trabajadores, lo que coincide con el Plan Nacional del Buen Vivir. Las políticas públicas no han sido consideradas de largo plazo y para ello se han creado estrategias propias de educación especializada en la familia y en contratación de personal técnico en áreas de desarrollo de la organización.
\end{abstract}

\section{Palabras clave}

Política pública, empresa familiar, fomento estatal, fomento empresarial.

* Ecuatoriano. Máster en Estadística Aplicada. Universidad Técnica de Ambato. Docente titular agregado.

** Ecuatoriano. Doctor en Ciencias Empresariales. Universidad Técnica de Ambato. Subdecano de la Facultad de Ciencias Administrativas.

*** Ecuatoriano. Máster en Producción Agroindustrial. Universidad Técnica de Ambato. Docente. 


\begin{abstract}
The policy of promoting businesses is looks for exploiting the potential of the country, the objective of this study was: To determine the use of public policies in the growth of the family business. The descriptive research was applied to two hundred and twelve family businesses and the results found were: Family businesses are run by family members, especially the founder, with a capacity to improve the quality of life of their workers, which coincides with the National Plan for Good Living. Public policies have not been considered of long term and for this specialized educational strategies in family have been created and in recruitment of technical staff in areas of organizational development.
\end{abstract}

\title{
Keywords
}

Public policy, family business, State development, business development.

Forma sugerida de citar: Santamaría Freire, Edwin, Moreno Gavilanés, Klever, \& Santamaría Díaz, Edwin (2016). Aprovechamiento de la política pública en el fomento de la empresa familiar. Revista Retos, 11(1), pp.27-40

\section{Introducción}

Ecuador ha puesto en marcha el Plan Nacional del Buen Vivir, a partir de lo cual se desprenden políticas que buscan generar bienestar en la sociedad. Al ser un modelo económico orientado por el socialismo del siglo XXI, este modelo difiere de los modelos de otros países de la región, porque crea condiciones y comportamientos diferentes de las empresas familiares, que buscan aprovechar las políticas públicas existentes, estableciendo relaciones entre las políticas públicas y el desarrollo de la planificación o gestión que hacen las familias propietarias para convertir las leyes en una oportunidad de crecimiento.

El estudio científico de la empresa familiar es planteado como la evolución desde el modelo de los Dos Círculos de Lansberg, Modelo de los Tres Círculos de Tagiuri y Davis y el Modelo de Desarrollo de la Empresa Familiar de Gersick, Davis, Hampton y Lansberg, revisados en (Santamaría, 2014), que ha permitido identificar los nexos que existen entre la empresa, familia y sociedad, haciendo necesario la medición del aporte del estado en el crecimiento de las empresas familiares que nacen desde las interacciones entre los elementos del sistema mismo.

Por lo que se plantea como pregunta de investigación: ¿Las políticas públicas promueven el desarrollo de las empresas familiares?, donde el análisis se centra en:

- Participación en programas de capacitación y formación. 
- Obtención de financiamiento y garantías del estado.

- Aplicación de programas de mejoramiento de procesos productivos.

- Participación en programas de mejoramiento en la gestión administrativa.

La política de estado definida "Como un curso de acción de la gestión pública que institucionaliza la intervención pública en respuesta a un problema social identificado como prioritario, y que se convierte de esta manera en materia de política de Estado" (Andino, 2013, p. 32). En este sentido, el papel central que juega el Estado para llevar a cabo las políticas públicas enmarcándolas en lograr la integración y el equilibrio en este caso de la familia, empresa y sociedad.

De acuerdo al Presidente de la República: "Existe gran potencial de exportación en sectores como: plásticos, tubería, bombas, línea blanca, cables y transformadores, y reducción de importaciones en: farmacéuticos, agroquímicos y equipos para agro" (Presidencia de la República, Ecuador, 2015). De esto se ve el incentivo del Gobierno Central para la creación y desarrollo de las empresas en dichos sectores. Sin embargo, en el mismo informe, se menciona que el empresario debe asumir responsabilidades con la sociedad y de ello se han generado compromisos de la empresa con la ciudadanía.
Pro Ecuador indica que los incentivos a las empresas en el país son: "Deducción adicional del 100\% de los gastos incurridos para el cálculo del Impuesto a la Renta, capacitación técnica, investigación e innovación, estudios de mercado y competitividad, viaje y promoción comercial para apertura de nuevos mercados" (Instituto de Promoción de exportación e inversiones, Ecuador, 2015), esto hace que una política pública genere incentivos para la creación de empresas.

\section{Política pública}

La política pública se conoce como "El conjunto de actividades de las instituciones de gobierno, que actúan de manera directa o a través de agentes, y que van dirigidas a tener una influencia determinada sobre la vida de los ciudadanos" (Barreiro, Berro, Romano, \& Menéndez, 2013, p. 45). Así mismo "Una política pública se define como una decisión caracterizada por una conducta consistente y repetitiva por parte de aquellos que la elaboran como de aquellos que se atienen a ella" (Ruiz Sánchez, 2002, p.18). Es decir que la política pública busca la participación de los involucrados para la creación de la normativa que permita su desarrollo consistente con las necesidades de la población (Solarte Pazos, 2004, p. 35) lo resume como: "Aquello que un Gobierno hace o deja de hacer". Por su parte define (Olano García, 2006, 
p. 139) como "Un conjunto organizado de disposiciones que configura y ordena los poderes del Estado por ella construidos".

El Plan Nacional del Buen Vivir menciona que "El tercer eje estructurante es la transformación del sistema económico para que se convierta en un sistema social y solidario, en el que converjan la economía de mercado, la economía pública y la economía popular y solidaria" (Subsecretaría de Planificación Nacional Territorial y Políticas Públicas, Ecuador, 2013). En los objetivos dentro del eje se observa la consolidación del sistema económico social y solidario, la transformación de la Matriz Productiva, la eficiencia en sectores estratégicos que puede ser aprovechado como política por las empresas familiares.

La Estrategia Nacional para el cambio de la Nueva Matriz Productiva se implementa mediante herramientas de planificación, acciones públicas, definidas por distintos niveles de gobierno y por acciones privadas (Vicepresidencia de la República, del Ecuador, 2013). Apoyadas en Ministerios que ejecutan las leyes vigentes, con orden y prioridad de acción en todos los organismos del estado.

El Gobierno ecuatoriano da "Prioridad a la dotación al sector público y privado, de herramientas que permitan consolidar las condiciones requeridas para atraer a los inversionistas" (Glas Espinel, Pastor, Albor- noz, Guerrero \& Solís, 2012, p. 07). Para ello, "El Plan Nacional del Buen Vivir está acompañado por un sistema de monitoreo y evaluación que hará posible conocer los impactos de la gestión pública" (Subsecretaría de Planificación Nacional Territorial y Políticas Públicas del Ecuador, 2013).

Para las empresas el aprovechamiento de los impuestos es la obtención de beneficios que nacen desde "Donde no hay contraprestación directa alguna por parte del ente público que lo exige" (Muñoz Rodríguez, 2013, p. 08) en este caso para la empresa familiar.

\section{Empresas familiares}

Una empresa familiar es considerada como aquella que la propiedad y las decisiones administrativas están dadas por los miembros de un grupo familiar, criterio que es compartido por (Corona, 2005), (Rueda Rodríguez, 2008), (Gil Estallo, 2010), además, (Casillas Bueno, Díaz Fernandez, Rus Rufino \& Vázquez Sánchez, 2014, p. 128) "La planificación estratégica en la empresa familiar requiere la consideración de aspectos propios de la empresa e inclusión de elementos como: La visión de la familia empresaria, las políticas del estado, incentivos a la empresa y protección familiar".

La planeación estratégica (Serna \& Suárez, 2005, p. 17): "Es el proceso mediante el cual quienes toman decisiones en una organización obtienen, 
procesan y analizan información pertinente, interna y externa", es decir, la empresa familiar buscará desarrollar y proteger la interdependencia familia-empresa que garanticen el patrimonio y desarrollo empresarial.

Las empresas familiares realizan el proceso de planificación estratégica como un reto de integrar los dos sistemas (familia-empresa), con una clara orientación hacia el crecimiento de la empresa (Acosta \& Paula, 2010).

Así mismo, se conoce dentro de la provincia de Tungurahua que la empresa familiar es predominante entre las empresas, siendo el 95,4\% empresas de estas características, es decir, que el propietario de la empresa es quien administra y en varias ocasiones esta acompañado por otro miembro de la familia (Santamaría \& Pico, 2015).

Se observa que las empresas familiares pueden aprovechar la política pública para el mejoramiento de las condiciones de trabajo desde la capacitación y formación de temas específicos de mercado y producción, participación en programas y obtención de financiamiento que potencializan a la organización, de acuerdo a ello se plantea como hipótesis de trabajo: Las empresas familiares participan activamente en programas de fomento de la producción.

\section{Metodología}

Para el desarrollo de la investigación se utiliza el positivismo, don- de "Todo conocimiento es válido si está basado en la observación de los hechos sensibles" (Inche M., y otros, 2003, p. 30). Además, "La metodología adoptada sigue el modelo hipotético-deductivo de las ciencias naturales, por medio de categorías de los fenómenos sociales en variables 'dependientes' e 'independientes', entre las que se establecen relaciones estadísticas" (Ricoy Lorenzo, 2006, p.16). El trabajo se basa en la observación de la realidad y evaluada estadísticamente, con lo cual se crea evidencia que demuestre la relación de las variables dentro de la hipótesis formulada.

Para la investigación se utiliza como población de estudio a las empresas familiares de la provincia de Tungurahua en Ecuador, donde “existen 58721 hogares en el área urbana, según información obtenida del censo realizado en el año 2010, existen 24324 empresas que fueron consideradas en el censo económico del mismo año" (Santamaría, 2014, p.14) por lo cual es un referente de cantidad de empresas en relación a las familias existentes en el territorio. Para la muestra se considera el 94\% de nivel de confianza, esta proporción se debe a que las empresas familiares limitan el acceso a la información.

Se consideró para el estudio a doscientos doce empresas que reúnan las condiciones de empresas familiares, es decir, "Aquella organización donde los miembros de una familia son el eje 
central para la gestión, dirección y funcionamiento de la misma, haciendo que los roles de familia y empresa se mezclen en dinámicas propias y difíciles de entender por separado" (Santamaría \& Pico, 2015, p.122).

Se aplica la encuesta como instrumento de recolección de información por considerarse como, "El instrumento de obtención de información, basado en la observación y el análisis de respuestas a una serie de preguntas" (Inche M., y otros, 2003, p. 34) Es decir, este instrumento es el medio adecuado para sintetizar la realidad observada en bases de datos.

Se aplica encuesta piloto a treinta y cinco miembros de la carrera de Organización de Empresas, quienes fueron seleccionados bajo el criterio de que posean al menos un familiar como propietario de una empresa. Se considera como válida la muestra piloto por ser un referente de las empresas familiares y por tener conocimiento teórico de ciencias administrativas. Al resultado se le aplica el Alfa de Cronbach obteniendo el 0.807, es decir, la encuesta posee validez para la recolección de información.

El instrumento utiliza variables cualitativas y para la medición escalas ordinales y nominales, con preguntas relacionadas a: propiedad de la empresa, gestión administrativa, conocimiento y participación en programas de fomento empresarial y efectos de la política pública en la sociedad. Los ítems fueron validados desde el contenido teórico por expertos en administración pública y administración de empresas familiares, posteriormente, presentado a cinco empresarios que avalaron los cuestionamientos presentados, es decir, el instrumento posee objetividad.

La aplicación del instrumento bajo la técnica de autollenado se aplicó en junio 2015 a un representante de la empresa y miembro de la familia propietaria, siempre dentro de la empresa estudiada, de modo que haya representatividad en el estudio. La muestra se formó con empresas de la provincia de Tungurahua, que fueron seleccionadas aleatoriamente de los registros del Consorcio de Cámaras de Producción del Tungurahua, siempre y cuando posean la característica de empresa familiar.

El procesamiento de la información se realizó por medio del programa SPSS 21.0 el mismo que permite procesar la información y presentar tablas y gráficos descriptivos, además de contrastes de hipótesis.

Para la medición de confiabilidad de los resultados se utiliza el test de Kruskal Wallis y análisis post hoc por medio de HSD de Tukey, permitiendo encontrar diferencias significativas entre los grupos de estudio y contrastar la hipótesis de trabajo: Las empresas familiares que conocen sobre los programas de fomento de la producción participan activamente en los mismos. 


\section{Resultados}

De los datos recopilados, en la Fi- propiedad de la empresa que es recogura 1 se observa la pregunta de la nocida como patrimonio de la familia.

Figura 1. Propiedad de la empresa

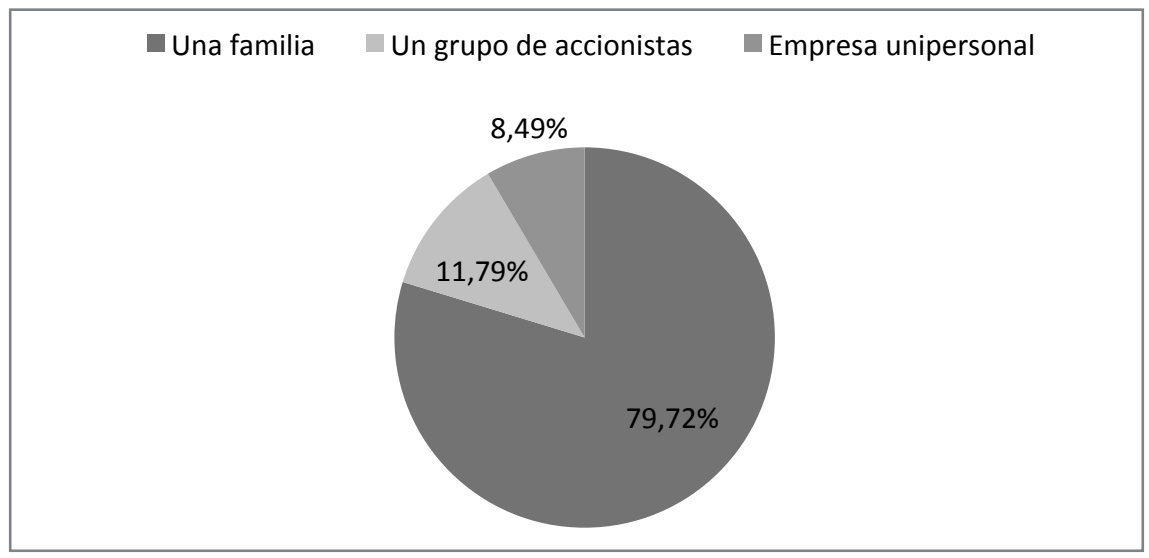

Fuente: Elaboración propia

La figura 2 muestra la respuesta gestión está a cargo de un miembro a la pregunta del encargado de la ges- de la familia y en un $13,27 \%$ le cotión administrativa de la empresa e rresponde a una persona externa a la indica que un $86,73 \%$ de los casos, la organización.

Figura 2. Encargado de la administración de la empresa familiar

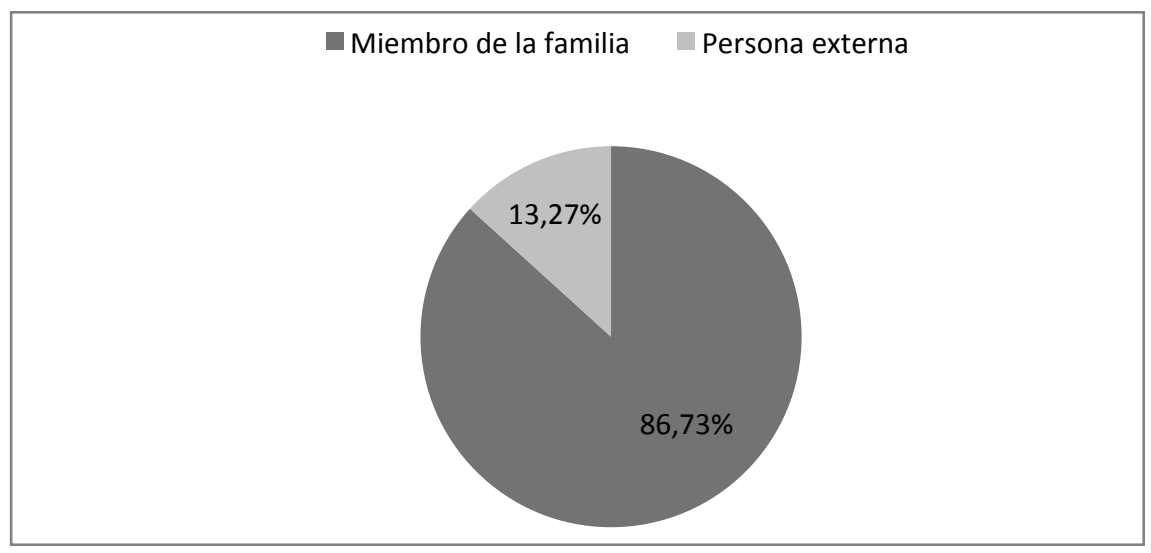

Fuente: Elaboración propia 
Como lo muestra la Figura 3, de del administrador, el 56\% posee un la pregunta del nivel de formación título terminal y de posgrado.

Figura 3. Nivel de Formación del administrador de la empresa familiar

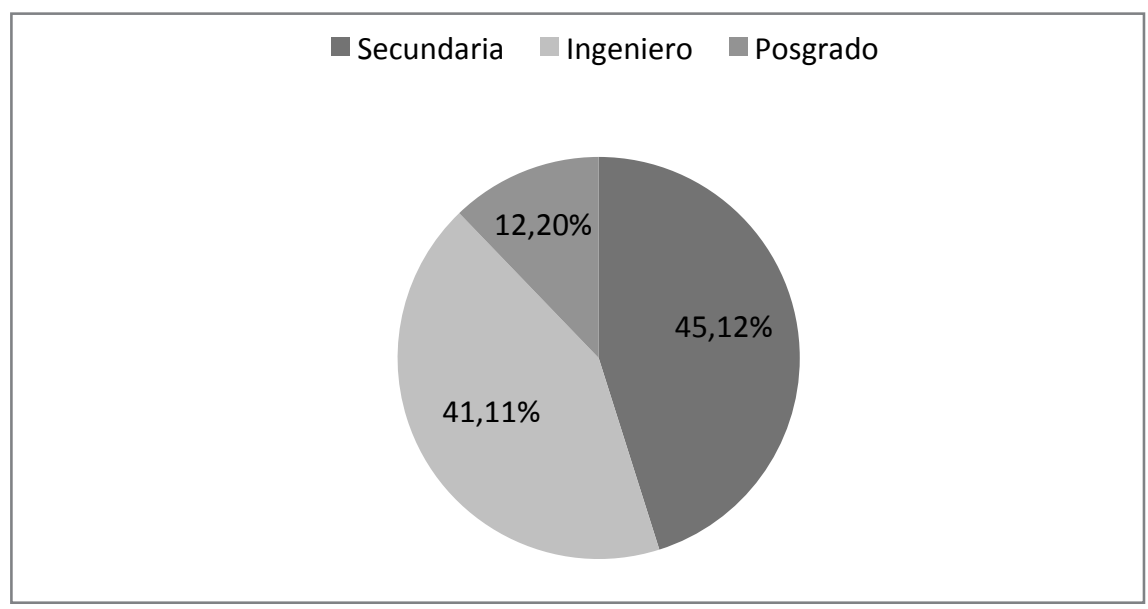

Fuente: Elaboración propia

Se compara el conocimiento de los programas y la participación en los mismos por parte de las empresas familiares, para ello, se realiza el test de Kruskal Wallis, por tratarse de variables no paramétricas (cualitativas), de ello se desprende la Tabla 1, donde se observa que hay diferencias en los rangos promedio, y el contraste de hipótesis arroja $\mathrm{p}$-value $=0,000$ es decir, hay evidencia estadísticamente significativa a nivel de confianza del $99 \%(\alpha=0,01)$ de la existencia de una relación entre el nivel de conocimiento y la participación en los programas de política pública por parte de la empresa familiar. 


\section{Tabla 1. Resultados de Test Kruskal Wallis}

\begin{tabular}{|c|c|c|c|}
\hline & $\begin{array}{l}\text { Ha participado de los programas } \\
\text { de fomento del Gobierno }\end{array}$ & $\mathrm{N}$ & Rango promedio \\
\hline \multirow{5}{*}{$\begin{array}{l}\text { Conoce de los } \\
\text { programas de fomento } \\
\text { a la empresa }\end{array}$} & Capacitación & 55 & 75,65 \\
\hline & Financiamiento & 63 & 106,98 \\
\hline & Gestión Administrativa & 47 & 118,53 \\
\hline & Mejoramiento de procesos & 30 & 87,95 \\
\hline & Total & 195 & \\
\hline
\end{tabular}

Fuente: Elaboración propia

En el análisis post hoc realizado por medio de HSD Tukey se encuentran grupos homogéneos como se muestra en la Tabla 2.

Tabla 2. Resultados de Test HSD Tukey

\begin{tabular}{|c|c|c|c|c|}
\hline & \multirow{2}{*}{$\begin{array}{l}\text { Conoce de los programas de } \\
\text { fomento a la empresa }\end{array}$} & \multirow{2}{*}{$\mathrm{N}$} & \multicolumn{2}{|c|}{$\begin{array}{l}\text { Subconjunto para } \\
\text { alfa }=0.05\end{array}$} \\
\hline & & & 1 & 2 \\
\hline & No conozco de estos programas & 39 & 1,54 & \\
\hline & Sí conozco la publicidad & 38 & 1,61 & \\
\hline \multirow{3}{*}{$\begin{array}{l}\text { HSD de } \\
\text { Tukey }\end{array}$} & Sí he participado & 58 & & 2,33 \\
\hline & $\begin{array}{l}\text { Sí he acudido a la institución para } \\
\text { informarme }\end{array}$ & 77 & & 2,42 \\
\hline & Sig. & & 0,991 & 0,980 \\
\hline
\end{tabular}

Fuente: Elaboración propia 
Se presenta el análisis del uso de los programas de gobierno y la percepción de las empresas familiares.

Figura 4. Impacto de la política pública

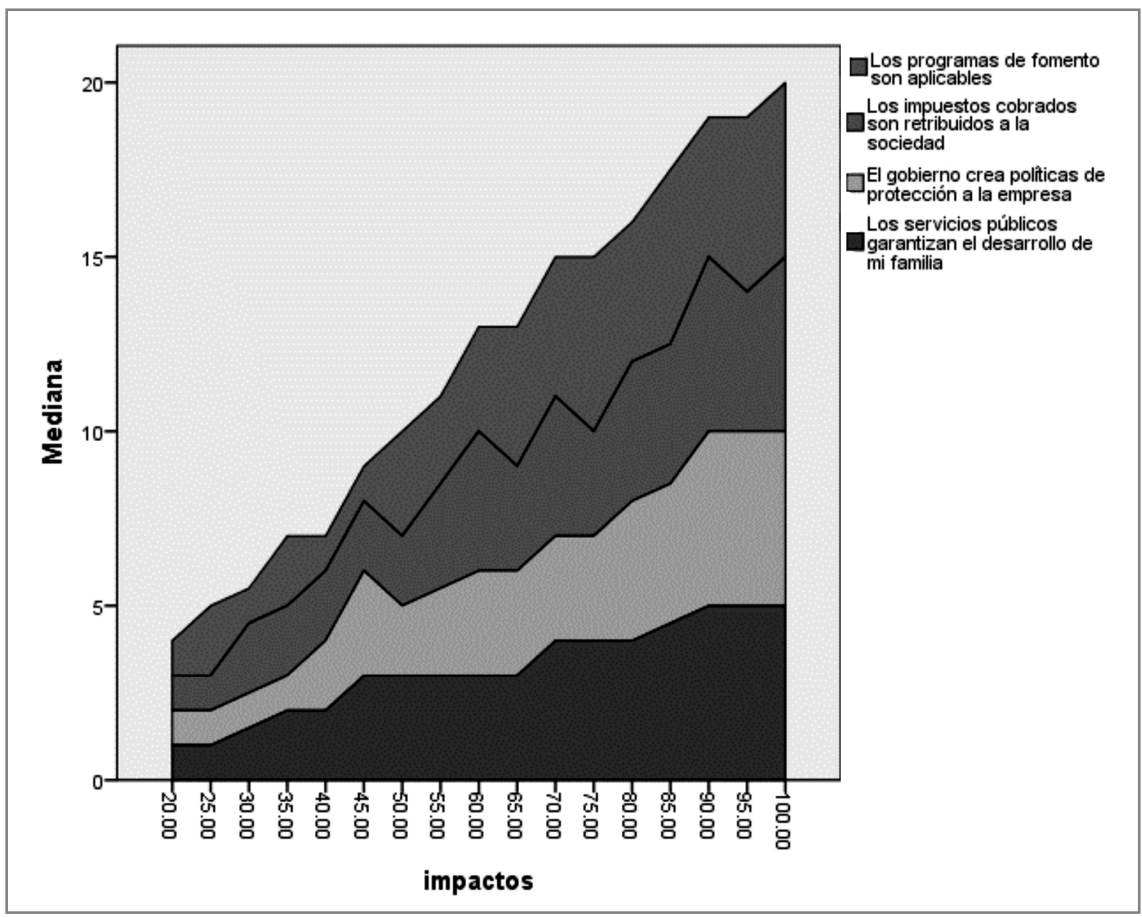

Fuente: Elaboración propia

Se realiza reducción de dimensiones por correspondencias principales entre la participación en programas de fomento del gobierno y la inversión requerida para su cumplimiento, de lo cual se reduce a dos di- mensiones con inercia de 0.294, por lo cual se demuestra confiabilidad por medio de en test de chi cuadrado, es decir que, que hay evidencia estadísticamente significativa que las variables están relacionadas entre sí. 
Figura 5. Dimensionamiento de la participación e inversión de la empresa familiar

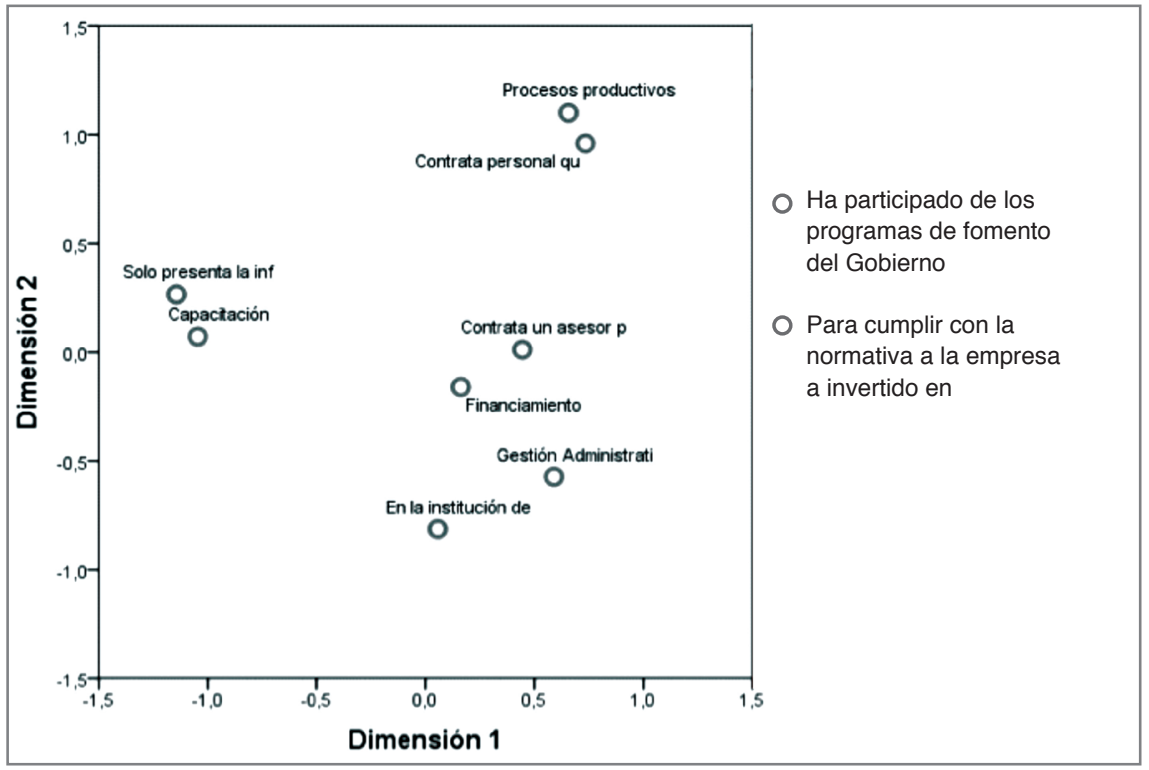

Fuente: Elaboración propia

\section{Discusión}

En la Figura 1, el 79.72\% menciona que es de propiedad directa de la familia, de lo cual se puede observar que las empresas familiares están bajo el liderazgo autocrático sin participación de la familia en la toma de decisiones, esto desde la estructura de personería jurídica y roles de participación dentro del hogar. Haciendo que la decisión de aprovechar la política pública sea casi exclusivamente del fundador de la empresa.

Los propietarios se encargan de dirigir a la empresa, Figura 2, además que buscan especializarse en di- rección de empresas, esto como una estrategia formativa dentro de las familias para mantener el control y optimizar el uso de recursos disponibles, Figura 3. A medida que se integran las nuevas generaciones el nivel de educación aumenta, haciendo que las empresas se preparen para asumir su rol dentro de la sociedad.

Las empresas familiares participan activamente y buscan aprovechar los programas de fomento, ( $\mathrm{Ta}$ bla 1). Además que la divulgación de las políticas incrementa la participación. De ello se observa en la Tabla 2, dos grupos: Un primer grupo de 
quienes solo logran un conocimiento publicitario y un segundo grupo mayoritario formado por quienes acuden a las instituciones y participan en los programas.

El efecto de la política pública en las empresas familiares es analizado en la Figura 4, donde se cuestiona sobre la aplicabilidad de los programas, el uso de los impuestos para la sociedad, existencia de protección e incentivo a la empresa y garantía sobre la empresa familiar a lo cual, las empresas familiares prefieren crear estrategias propias para el cuidado patrimonial y crecimiento de las familias empresarias sin importar las políticas públicas existentes.

En la Figura 5, se encuentran relaciones entre: 1) Las empresas que desean mejorar sus procesos productivos invierten en personal capacitado para las nuevas actividades, 2) Las personas que solo presentan la información básica y utilizan capacitación brindada por el estado, y 3) Las empresas que contratan asesores o solo utilizan personas de la institución que participan en programas de financiamiento y mejoramiento administrativo. Indicando que a mayor es el aprovechamiento, las empresas familiares incrementan la percepción del impacto de las políticas públicas en las empresas y sociedad. Así mismo las empresas que buscan el mejoramiento productivo evidencian mejor predisposición para acep- tar políticas públicas y contribuir a través del pago de tributos que beneficien a la sociedad.

\section{Conclusiones}

Las empresas familiares se consideran a aquellas organizaciones donde la propiedad y la gestión está a cargo de una familia o persona, sin importar que su estructura jurídica sea como empresa unipersonal o sociedad anónima. Concepción similar a lo indicado por (Casillas Bueno, Díaz Fernández, Rus Rufino \& Vázquez Sánchez, 2014) específicamente a lo relacionado a las características de empresa familiar.

La política pública de fomento de la empresa privada se reconoce como el conjunto de normativas, leyes y reglamentos que incentiva la creación, desarrollo y crecimiento de la empresa, (Andino, 2013) reconociendo que en Ecuador la misma está direccionada desde el Plan $\mathrm{Na}$ cional del Buen Vivir, de ahí que se puede comparar con los resultados obtenidos, donde las empresas familiares buscan prepararse para asumir su rol en la sociedad, donde se mezclan aspectos de familia y gestión administrativa.

La empresa familiar aprovecha las políticas públicas en el crecimiento de la empresa, siguiendo los lineamientos del Plan Nacional de Buen Vivir (Subsecretaría de Planificación Nacional Territorial y Políti- 
cas Públicas, Ecuador, 2013) a través de: Capacitación, financiamiento, asesoramiento en procesos y gestión administrativa de las empresas, sin embargo, la planificación familiar de patrimonio se considera como una estrategia independiente de la política pública, esto similar a lo indicado por organismos públicos como Senplades y Pro Ecuador.

El aprovechamiento de las políticas públicas en las familias empresarias acoge como estrategia la educación especializada en Administración de sus miembros y al mismo tiempo para el mejoramiento de procesos se contrata personal con formación en el área específica.

Las empresas familiares están desarrollándose acorde a los principios del Plan Nacional del Buen Vivir, es decir que se aprovechan los incentivos a la producción y generan fuentes de empleo que mejoran la calidad de vida de la ciudadanía, que concuerda con la política pública como lo manifiesta (Subsecretaría de Planificación Nacional Territorial y Políticas Públicas del Ecuador, 2013).

Se reconocen dos grupos de empresas familiares, el primero que busca obtener conocimiento de los programas de Gobierno sin participar, y el segundo grupo que participa en programas de capacitación hasta el desarrollo de procesos que promue- ven la productividad y desarrollo de la empresa.

La especialización en dirección de empresas se da como una estrategia formativa dentro de las empresas familiares. Que concuerda con lo indicado por (Acosta \& Paula, 2010) referente al direccionamiento de las empresas familiares.

(Corona, 2005) La empresa familiar gestiona y controla su patrimonio. Aspecto que coincide con los resultados obtenidos, donde la familia toma sus propias estrategias para el cuidado patrimonial y crecimiento de las familias empresarias sin importar las políticas públicas existentes.

Las políticas públicas aplicables para la empresa familiar, sin embargo, dichas políticas no son consideradas que en el largo plazo vayan a crear beneficios para la protección y desarrollo de sus propietarios, por lo tanto, las familias empresarias generan sus propias estrategias de crecimiento.

Las familias están dispuestas a utilizar las políticas públicas en beneficio de la empresa, para ello realizan acciones estratégicas como: Contratación de personal y formación de los administradores de la empresa. Con ello se consigue que haya fuentes de trabajo y crecimiento empresarial. Acciones que están relacionadas con el Plan Nacional del Buen Vivir.

Los aportes del estado hacia el desarrollo de la empresa familiar se 
basan en la creación de planes de capacitación, asesoramiento y financiamiento lo cual permite respaldar su actividad productiva. A partir de lo cual se pueden plantear nuevas líneas de investigación como el valor agregado que genera la política pública en la creación de riqueza de dichas empresas.

\section{Bibliografía}

Acosta, A. M., \& Paula, P. U. (2010). La planificación estratégica en las empresas familiares. Revista del Centro de Investigación de Ciencias Administrativas y Gerenciales, 81-87.

Andino, V. (2013). Políticas públicas para la economía social y solidaria: Caso de estudio de Ecuador. Quito: RELIESS.

Barreiro , F., Berro , M., Romano , C., \& Menendez, W. (2013). Políticas públicas. Guía didáctica. Uruguay: Diseño Editorial.

Casillas Bueno, J. C., Díaz Fernandez, M. D., Rus Rufino, S. I., \& Vázquez Sánchez, A. (2014). La gestión de la empresa familiar. Conceptos, casos y soluciones. 2. ${ }^{a}$ edición. Madrid: Ediciones Paraninfo.

Corona, J. (2005). Manual de la empresa familiar. Madrid: Ediciones Deusto.

Gil Estallo, M. d. (2010). Cómo crear y hacer funcionar una empresa. Madrid: ESIC Editorial.

Glas Espinel, J., Pastor, W., Albornoz, E., Guerrero, J., \& Solís, W. (Enero de 2012). Sectores estratégicos. Fonte: http://www.sectoresestrategicos. gob.ec/wp-content/uploads/downloads/2012/08/CatalogoDeInversion2012-Espanol_Parte1.pdf

Inche M., J., Andía C., Y., Huamanchumo
V., H., López O., M., Vizcarra M., J., \& Flores C., G. (2003). Paradigma cuantitativo: un enfoque empírico y analítico. Industrial Data, vol. 6(n. 1), 23-37.

Instituto de Promoción de exportación e inversiones, Ecuador (2015). Pro Ecuador. Fonte: Reglas e incentivos para la inversión: http://www.proecuador.gob.ec/invierta-en-ecuador/porque-invertir-en-ecuador/ reglas-claras-e-incentivos-para-lainversion/

Muñoz Rodríguez, R. (2013). Impuesto de sociedades. Madrid: Ministerio de Educación, Cultura y Deporte.

Olano García, H. A. (2006). ¿Qué es una Constitución? Dikaion: Revista de Actualidad Jurídica, 15, 136-153.

Presidencia de la República, Ecuador (18 de Abril de 2015). Enlace Ciudadano 420. Incentivo económico a sectores productivos. San Isidro de Puengasí, Pichincha, Ecuador: Presidencia.

Ricoy Lorenzo, C. (2006). Contribución sobre los paradigmas de investigación. Educação. Revista do Centro de Educação, vol. 31 (n. 1), 11-22.

Rueda Rodríguez, J. (2008). Un enfoque múltiple de la economía española: principios y valores. 175 opiniones de los principales investigadores de España. Madrid: Ecobook- Editorial del Economista.

Ruiz Sánchez, C. (2002). Manual para la elaboración de políticas públicas. México: Plaza y Valdes.

Santamaría, E. (2014). La estructura de la familia empresaria y las repercusiones en la sucesión de las empresas de la provincia de Tungurahua, Ecuador. 13th Summer Institute of the PRSCO 2014 (pp. 1-20). Loja: Universidad Técnica Particular de Loja. Fonte: http://issuu.com/ edwinsantamaria/docs/la_estructu- 
ra_de_la_familia_empresa

Santamaría, E., \& Pico, F. (2015). Empresas familiares: Análisis de los factores estratégicos que influyen en la dinámica familia-empresa. Revista Politécnica, 35(2), 113-123.

Serna, H., \& Suárez, E. (2005). La empresa familiar. Estrategias y herramientas para su sostenibilidad y crecimiento. Bogotá: Editorial Temis.

Solarte Pazos, L. (2004). Las evaluaciones de políticas públicas en el estado liberal. Colombia: Programa Editorial Universidad del Valle.
Subsecretaría de Planificación Nacional Territorial y Políticas Públicas, Ecuador (2013). Plan Nacional para el Buen Vivir 2013-2017. Quito: SENPLADES.

Vicepresidencia República, Ecuador (24 de Mayo de 2013). Vicepresidencia República del Ecuador. Fonte: Ecuador ama la Vida : http://www.vicepresidencia.gob.ec/objetivo-generalobjetivos-estrategicos-y-entornopara-el-cambio-2/

Recepción: 12-10-2015 - Aceptación: 21-06-2016 